\title{
Rimozione dei contaminanti ambientali mediante applicazione di processi ultrasonici ed adsorbimento su carboni attivi: una tecnologia innovativa
}

\author{
Roberta Amendola ${ }^{1}$ \\ ${ }^{1}$ Affiliation not available
}

\begin{abstract}
La rimozione di contaminanti ambientali dal nostro sistema idrico continua ad essere prioritaria, in quanto tali sostanze hanno impatti negativi sull'ambiente e sulla salute umana. Tra le tecniche più diffuse per la rimozione di materia organica presente nelle acque reflue, vi sono i processi di ossidazione avanzata, ed in particolare l'adsorbimento mediante carbone attivo granulare. Studi recenti hanno scoperto come l'utilizzo di processi ad ultrasuoni (US) migliori l'adsorbimento per molti contaminanti ambientali. Nel presente lavoro vengono descritte le indagini condotte per esaminare e valutare l'efficacia del processo di adsorbimento del fenolo, scelto come indicatore di materia organica, mediante carbone attivo granulare (GAC), in assenza e in presenza di ultrasuoni. I risultati ottenuti dimostrano come l'utilizzo combinato di carbone attivo granulare e di ultrasuoni migliori l'adsorbimento del fenolo, in quanto garantisce un tasso di adsorbimento più elevato e una maggiore rimozione della sostanza organica. Ulteriori sviluppi scientifici, inoltre, danno conferma di come l'impiego di irradiazioni ultrasoniche combinate con l'azione del carbone attivo granulare, e con diverse tecnologie, migliori drasticamente la rimozione di contaminanti ambientali dalle acque reflue, garantendone la depurazione e il riutilizzo.
\end{abstract}

\section{Problemi e soluzioni nella rimozione di conta- minanti ambientali}

Lo sviluppo tecnologico, spesso non in linea con le politiche di sviluppo sostenibile ${ }^{1}$, continua ad alimentare la presenza di contaminanti emergenti nel nostro sistema idrico, ma l'attuale sistema di trattamento delle acque reflue (Fig. 1) non è efficace nella rimozione delle diverse classi di tali contaminanti ${ }^{2}$. 


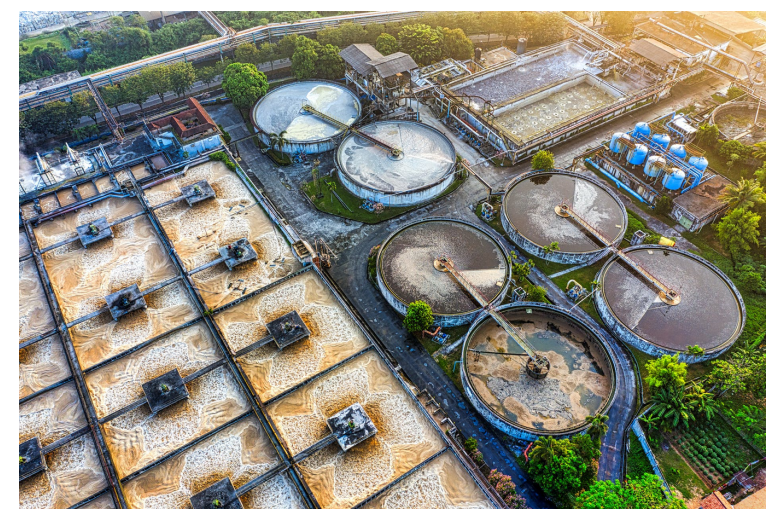

Figure 1: Impianto di trattamento delle acque reflue (fonte: Pexels)

Per la rimozione di tali sostanze, che comprendono, tra le altre, prodotti farmaceutici (Fig. 2), prodotti per la cura della persona, o additivi industriali, sono necessarie tecniche di rimozione più avanzate al fine di depurare e riutilizzare le acque reflue ${ }^{3}$. Tra le sostanze considerate come inquinanti prioritari, vi sono il fenolo ed i suoi derivati, presenti negli effluenti di industrie petrolchimiche o farmaceutiche, e fortemente dannosi per l'ambiente e per la salute umana ${ }^{4}$.

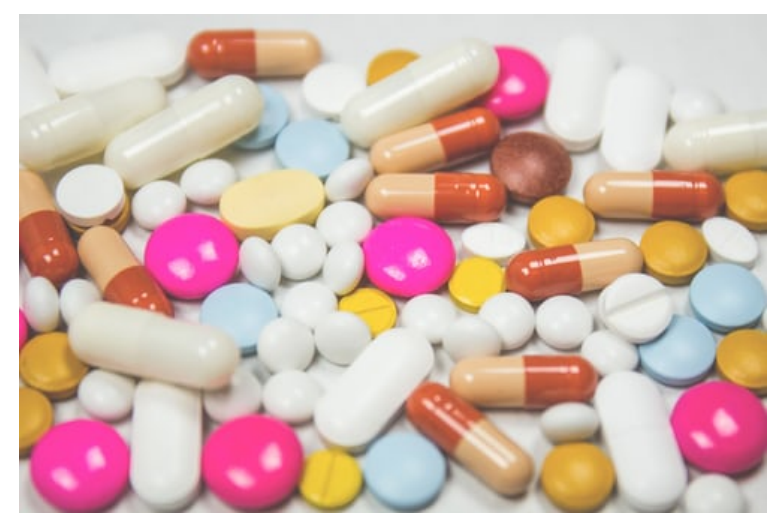

Figure 2: Una delle principali cause della presenza dei contaminanti emergenti nelle acque reflue: i prodotti farmaceutici (fonte: Unsplash)

In letteratura sono presenti innumerevoli tecniche per la rimozione del fenolo, che comprendono trattamenti avanzati, come l'ozonizzazione, trattamenti biologici e trattamenti enzimatici ${ }^{4}$. La tecnologia più diffusa per la rimozione di contaminanti ambientali comprende i processi di ossidazione 
avanzata (AOPs) ${ }^{5}$ ed in particolare l'adsorbimento mediante carbone attivo granulare (GAC). Studi precedenti hanno mostrato interesse verso un trattamento innovativo basato sulla rimozione di sostanze inquinanti, come i prodotti farmaceutici, attraverso l'uso di ultrasuoni (US) ${ }^{6}$ combinato con diverse tecniche, come la fotocatalisi ${ }^{7}$. L'utilizzo di ultrasuoni, quindi, combinato con tecniche di ossidazione avanzata ${ }^{8}$, migliora la rimozione di sostanze tossiche come il fenolo, grazie al fenomeno della cavitazione, ovvero la formazione e il successivo collasso delle cosiddette bolle di cavitazione.

Il presente lavoro si basa sullo studio condotto da ${ }^{9}$, che indaga sull'influenza degli ultrasuoni nell'adsorbimento del fenolo, scelto come indicatore di materia organica, mediante carbone granulare attivo. Le valutazioni sono state effettuate, sia considerando la sola azione del carbone granulare attivo, sia considerando la sua azione combinata con gli ultrasuoni. Il processo è stato implementato sia per soluzioni acquose che per acque reflue urbane al fine di poter valutare l'applicazione di tali trattamenti anche a larga scala. Nel lavoro si richiamano, inoltre, ulteriori studi che evidenziano come l'utilizzo degli ultrasuoni, combinati con diverse tecnologie, sia un valido alleato nella rimozione dei contaminanti ambientali e migliori il trattamento e la depurazione delle acque reflue.

\section{Rimozione dei contaminanti ambientali tramite US e GAC: il fenolo come sostanza modello}

I test sono stati condotti sia su soluzioni acquose che su campioni di acque reflue domestiche, prelevati dall'impianto di trattamento delle acque reflue di Salerno; in entrambi i casi la concentrazione di fenolo presente raggiunge un picco di $100 \mathrm{mg} / \mathrm{l}$. L'influenza della potenza degli ultrasuoni è stata testata a tre diverse densità: 1,06, 1,60 e 3,20 W/ml. In condizioni di bassa densità è stato riscontrato che le prestazioni degli ultrasuoni non hanno effetti significativi nella rimozione del fenolo e che, inoltre, il tempo di trattamento si prolunga; aumentando la densità i risultati ottenuti appaiono più significativi.

Sono stati effettuati test di adsorbimento, con carbone attivo granulare (GAC) mediante l'utilizzo di un agitatore magnetico, e test di adsorbimento con carbone attivo granulare (GAC) e ultrasuoni (US). Per questi ultimi, al fine di ottenere un'adeguata miscelazione all'interno del reattore, è stato sfruttato l'effetto idrodinamico dovuto al fenomeno della cavitazione. Per entrambi i test effettuati sono state garantite condizioni di miscelazione uniforme all'interno del reattore. Dai test effettuati su soluzione acquosa mediante il solo utilizzo del GAC, è emerso che il tasso di adsorbimento del fenolo aumenta fino al raggiungimento del tempo di equilibrio di $5 \mathrm{~h}$, per poi rimanere costante. Dai test combinati (US+GAC), emerge invece che il tempo di equilibrio viene raggiunto in sole $4 \mathrm{~h}$; inoltre il tasso di 
adsorbimento del fenolo è più alto. Effettuando gli stessi test sui campioni di acque reflue urbane, è emerso che il processo US+GAC è meno efficace in termini di rimozione del fenolo a causa della presenza di sostanze organiche naturali all'interno del refluo; tali sostanze, una volta adsorbite dal GAC, saturano i pori, limitando quindi l'adsorbimento del fenolo.

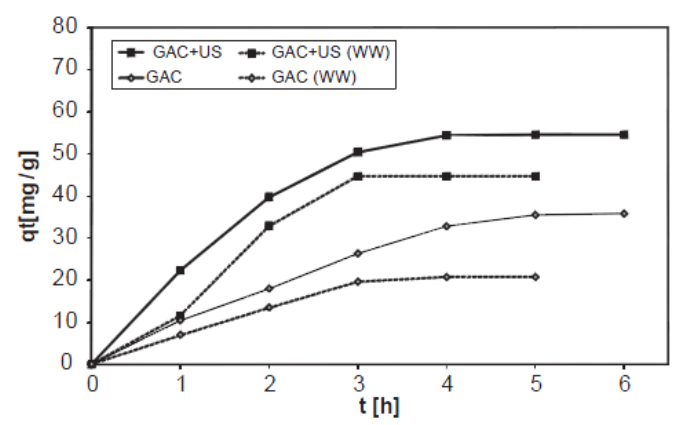

Figure 3: Curve cinetiche di adsorbimento del fenolo sia in soluzione acquosa che in acque reflue, con GAC e con GAC+US (riadattata da Landi et al., 2010)

Dallo studio delle curve cinetiche (Fig.3), emerge che il tasso di adsorbimento del fenolo è molto più alto in presenza di ultrasuoni, sia per la soluzione acquosa che per le acque reflue. L'effetto del trattamento US+GAC è stato studiato anche in termini di riduzione di TOC (Total Organic Carbon) e di COD (Chemical Oxygen Demand). La riduzione del TOC, dopo 5 ore di trattamento, da parte dei soli US è di circa l' $8 \%$, la riduzione per adsorbimento nel GAC è del $35 \%$ e quella per adsorbimento con US+GAC è superiore al $50 \%$. I trattamenti permettono, inoltre una significativa riduzione del COD: I'adsorbimento sui test GAC permette una riduzione del $25 \%$, mentre il processo combinato con l'irradiazione US permette una riduzione fino al $40 \%$. Inoltre, per la riduzione del COD, si può notare che dopo $3 \mathrm{~h}$ in adsorbimento solo in GAC, si raggiunge una condizione stabile. Nell'adsorbimento potenziato con irradiazione US, invece, la condizione stabile viene raggiunta dopo sole $2 \mathrm{~h}$ di trattamento. Quindi, il punto di equilibrio può essere raggiunto in un tempo inferiore con l'adsorbimento con gli US rispetto all'adsorbimento senza.

Questi risultati indicano come l'utilizzo degli ultrasuoni migliori l'adsorbimento del fenolo mediante carbone attivo granulare. L'efficacia di tale tecnica combinata è analizzata anche da ${ }^{10}$. In letteratura sono presenti ulteriori sviluppi nell'ambito dell'utilizzo degli ultrasuoni, combinati con diverse tecnologie, al fine di migliorare la rimozione di contaminanti ambientali nelle acque reflue. Lo studio condotto da ${ }^{11}$ ha mostrato come 
I'utilizzo di ultrasuoni a bassa frequenza riduca notevolmente la concentrazione di diclofenac nel refluo urbano; è emerso che i campioni trattati sono più biodegradabili e quindi l'irradiazione ultrasonica più essere utilizzata come pretrattamento seguito dal trattamento biologico. Lo studio condotto da ${ }^{12}$ dimostra come l'irradiazione ultrasonica migliori l'adsorbimento su carbone attivo mediante un nuovo processo ibrido che applica simultaneamente l'ultrafiltrazione della membrana, l'adsorbimento del carbone attivo e l'irradiazione degli ultrasuoni, rimuovendo, con elevata efficienza, i contaminanti emergenti. L'ultrafiltrazione su membrana è un'altra tecnica molto utilizzata per la depurazione delle acque reflue; le incrostazioni che possono formarsi sulla membrana costituiscono, però, uno dei principali inconvenienti per l'utilizzo di questa tecnologia. Si dimostra come combinando l'ultrafiltrazione su membrana con l'azione degli ultrasuoni, non solo è possibile controllare l'incrostazione della membrana, ma si migliora anche la performance del trattamento del refluo 13 14 14 . L'applicazione degli ultrasuoni può essere inoltre utilizzata per la rimozione di composti organici e inorganici da sedimenti contaminati; tale processo conduce ad ottimi risultati nell'ambito della rimozione dei contaminanti emergenti, sia come trattamento autonomo che come trattamento combinato con un processo elettrocinetico ${ }^{15}$. Ulteriori tecniche finalizzate alla rimozione di contaminanti emergenti mediante l'ausilio di ultrasuoni, combinati con ozonizzazione, sono analizzate in ${ }^{16} \mathrm{e}^{17}$.

\section{Conclusioni}

Nel lavoro è stata studiata l'applicazione degli ultrasuoni combinati con l'adsorbimento mediante carbone attivo granulare, al fine di migliorare ed implementare la rimozione dei contaminanti ambientali dal sistema idrico. Il fenolo è stato scelto come indicatore di materia organica e, per valutare le diverse rese di rimozione di tale sostanza, sono stati effettuati test di adsorbimento in GAC, in assenza e in presenza di ultrasuoni. I risultati ottenuti hanno mostrato come le irradiazioni ultrasoniche migliorino il processo di adsorbimento del fenolo mediante carbone attivo granulare. II processo combinato (US+GAC) garantisce infatti, sia per le soluzioni acquose che per il refluo urbano, un tasso di adsorbimento del fenolo più alto rispetto a quello ottenuto con la sola azione del GAC. È emerso però, che il solo trattamento mediante ultrasuoni a basse densità non porta a risultati significativi nella rimozione del fenolo. L'efficacia del processo combinato (US+GAC), è stata valutata anche in termini di rimozione di TOC e COD; i risultati ottenuti hanno mostrato come il trattamento combinato (US+GAC) abbia prestazioni superiori rispetto all'adsorbimento mediante il solo GAC. L'influenza degli ultrasuoni nel migliorare la resa di rimozione del fenolo mediante adsorbimento in GAC, è quindi molto forte. Ulteriori sviluppi scientifici hanno dato conferma di come l'utilizzo di irradiazioni 
ultrasoniche, combinato con diverse tecnologie di ossidazione avanzata, migliori nettamente la rimozione dei contaminanti ambientali dal nostro sistema idrico. Gli ultrasuoni costituiscono quindi, dei validi alleati per la depurazione e il riutilizzo delle acque reflue.

\section{References}

1.Naddeo, V., Belgiorno, V., Zarra, T. \& Scannapieco, D. Dynamic and embedded evaluation procedure for strategic environmental assessment. Land Use Policy 31, 605-612 (2013).

2.Bolong, N., Ismail, A. F., Salim, M. R. \& Matsuura, T. A review of the effects of emerging contaminants in wastewater and options for their removal. Desalination 239, 229-246 (2009).

3.M.Petrovic, D. B., S.Gonzalez. Analysis and removal of emerging contaminants in wastewater and drinking water. TrAC Trends in Analytical Chemistry 22, 685-696 (2003).

4.Villegas, L. G. C. et al.. A Short Review of Techniques for Phenol Removal from Wastewater. Current Pollution Reports 2, 157-167 (2016).

5.A.Cesaro, V. N. \& V.Belgiorno. Wastewater Treatment by Combination of Advanced Oxidation Processes and Conventional Biological Systems. Journal of Bioremediation \& Biodegradation 04, (2013).

6.Naddeo, V., Landi, M., Scannapieco, D. \& Belgiorno, V. Sonochemical degradation of twenty-three emerging contaminants in urban wastewater. Desalination and Water Treatment 51, 6601-6608 (2013).

7.V.Belgiorno et al.. Review on endocrine disrupting-emerging compounds in urban wastewater: occurrence and removal by photocatalysis and ultrasonic irradiation for wastewater reuse. Desalination 215, 166-176 (2007).

8. Water and wastewater disinfection by ultrasound irradiation - a critical review. Issue 3 16, 561-577 (2014).

9.M.Landi, V.Naddeo \& V.Belgiorno. Influence of ultrasound on phenol removal by adsorption on granular activated carbon. Desalination and Water Treatment 23, 181-186 (2010).

10. Cuomo, R. Metodi innovativi per il trattamento dei contaminanti emergenti: adsorbimento su carboni attivi combinati ad ultrasuoni doi:10.22541/au.157773067.72186856.

11.Naddeo, V., Belgiorno, V., Kassinos, D., Mantzavinos, D. \& Meric, S. Ultrasonic degradation mineralization and detoxification of diclofenac in 
water: Optimization of operating parameters. Ultrasonics Sonochemistry 17, 179-185 (2010).

12.Secondes, M. F. N., V.Naddeo, V.Belgiorno \& F.Ballesteros. Removal of emerging contaminants by simultaneous application of membrane ultrafiltration activated carbon adsorption, and ultrasound irradiation. Journal of Hazardous Materials 264, 342-349 (2014).

13.Naddeo, V., Borea, L. \& Belgiorno, V. Sonochemical control of fouling formation in membrane ultrafiltration of wastewater: Effect of ultrasonic frequency. Journal of Water Process Engineering 8, e92-e97 (2015).

14.L.Borea et al.. Wastewater treatment by membrane ultrafiltration enhanced with ultrasound: Effect of membrane flux and ultrasonic frequency. Ultrasonics 83, 42-47 (2018).

15.Fraiese, A. et al.. Ultrasonic processes for the advanced remediation of contaminated sediments. Ultrasonics Sonochemistry 67, 105171 (2020).

16.Barra, P. Trattamenti avanzati delle acque reflue per la rimozione di contaminanti emergenti doi:10.22541/au.158195300.04059015.

17.Scarpa, A. Contaminanti emergenti nelle acque reflue: una bomba da disinnescare. (2020) doi:10.22541/au.160315286.63088719/v1. 